\title{
Comparison of two commercial kits for identifying and biotyping Haemophilus parainfluenzae
}

\author{
M Warren, Z Ahmet, E Houang
}

\begin{abstract}
The Minitek system and the more recently introduced Micro Scan HNID panels for the identification and biotyping of $98 \mathrm{~V}$ dependent Haemophilus isolates were compared. Identical results were obtained for 77 isolates. The discrepancy in the results of ornithine and urease was accounted for mainly by the mismatching of the identification by the two kits. When 13 isolates of $H$ parainfluenzae with mismatched biotypes were re-examined, the results from Micro Scan correlated with $92 \%$ of those obtained by Christensen's urea broth and $100 \%$ by the ornithine test (Lab M); the corresponding figures for Minitek were $61 \%$ and $30 \%$, respectively. Micro Scan was easy to handle on the bench and results were ready on the same day. These results suggest that further work is required to assess these two systems for the biotyping of $H$ parainfluenzae.
\end{abstract}

Biotyping of clinical isolates of Haemophilus species was first described by Kilian in $1976 .{ }^{1}$ It has become important in the diagnosis and epidemiological study of Haemophilus infections. $H$ parainfluenzae is generally regarded as a commensal in the respiratory tract and may cause sporadic local or systemic infections. Commercially available kits are increasingly used nowadays by laboratories carrying out routine tests for the identification of bacteria. The Minitek system was recommended as a satisfactory alternative method in the biotyping of $H$ parainfluenzae. ${ }^{2}$ We studied the genital and respiratory carriage rates of different biotypes of $H$ parainfluenzae in men. ${ }^{3}$ We used the Minitek system and compared the results with Micro Scan HNID panels.

\section{Methods}

Isolates from urethral or throat swabs, which were Gram negative for pleomorphic coccobacilli, were tested for $\mathrm{X}$ and $\mathrm{V}$ factor dependency on Nutrient Agar (Oxoid), as described previously ${ }^{3}$ Single colonies of $\mathrm{V}$ dependent isolates were first subcultured on to chocolate agar plates (Oxoid) incubated overnight at $35^{\circ} \mathrm{C}$ with $5-7 \%$ carbon dioxide. The culture was then used to inoculate the Minitek system and the Micro Scan HNID panels. The Minitek system for the differentiation of Neisseria was used with the Neisseria broth as recommended (BBL Microbiology Systems,
Division of Becton Dickinson \& Co., USA). The following substrates or tests are included: ornithine, indole, urea, ONPG, nitrate, dextrose, mannitol, lactose, sucrose, galactose, maltose, glycerol, mannose, and xylose. The wells were inoculated and all except the ONPG well were overlaid with sterile oil and incubated at $35^{\circ} \mathrm{C}$ under ambient air in the Minitek humidor. Results were read the next day as described by Back and Oberhofer. ${ }^{4}$ The trays were reincubated for a further 24 hours after which the nitrate test was performed. The identification and biotyping of the isolates was done according to the method of Kilian. ${ }^{1}$ The Micro Scan HNID system was designed for rapid identification and detection of $\beta$ lactamase production in Neisseria spp, Haemophilus spp, Branhamella catarrhalis and Gardnerella vaginalis, and the biotyping of $H$ influenzae and $H$ parainfluenzae (American Hospital Supply Corporation, Sacramento, California, USA). The panels included the following substrates for conventional and chromogenic tests: indoxyl phosphate, reduction of nitrate and nitrite, glucose, sucrose, lactose, fructose, maltose, ONPG, urea, ornithine, indole, L-prolyl- $\beta$-naphthylamide, $\mathrm{N}$ - $\gamma$-L-glutamyl- $\alpha$-naphthylamide, $\quad \mathrm{N}-\alpha-$ benzoyl-DL-arginine- $\beta$-naphthylamide, $\mathrm{p}$ nitrophenyl- $\alpha$-D-glucoside, starch, and penicillin. A heavy suspension of the organism was made in the special inoculum broth supplied with the panel which was then inoculated and incubated for four hours. After incubation the results of the reactions were scored and the Biotype Codebook for HNID Panels (American MicroScan) was used to determine the identification and biotype of the individual isolates.

\section{Results}

Ninety eight consecutive isolates of Gram negative pleomorphic coccobacilli that were $\mathrm{V}$ dependent were further examined with the commercial kits. Similar identification was

Results of ornithine hydrolase and urease tests by each method on 13 isolates of $H$ parainfluenzae with discordant results

\begin{tabular}{llllllll}
\hline & \multicolumn{7}{l}{ Number of strains: } \\
\cline { 2 - 7 } & 7 & 1 & 1 & 1 & 1 & 2 \\
\hline Christensen's urea & + & - & - & + & + & - \\
Minitek urea & + & + & + & - & + & + \\
MicroScan urea & + & - & - & + & - & - \\
Lab M ornithine & + & + & - & - & + & + \\
Minitek ornithine & + & - & + & - & + & + \\
MicroScan ornithine & + & + & - & - & + & + \\
\hline
\end{tabular}

E Houang

Correspondence to: Dr E Houang 
obtained from both kits for 77 isolates: $H$ parainfluenzae type $1(\mathrm{n}=6)$, type $11(\mathrm{n}=55)$, type $111(\mathrm{n}=8)$, type IV $(\mathrm{n}=2)$, H influenzae type $111(\mathrm{n}=2)$, and $H$ paraphrophilus $(\mathrm{n}=4)$. Discordant biotypes were given on 16 isolates identified as $H$ parainfluenzae, and two isolates as $H$ influenzae. The Minitek system failed to identify three isolates, of which Micro Scan identified one as $H$ influenzae type IV and one as $H$ paraphrophilus. It failed to identify the third isolate.

Of the 16 isolates of $H$ parainfluenzae with mismatched biotypes, 13 were examined again with both kits. As the key tests that gave rise to variation in the results were ornithine and urea, a third method was used. An ornithine test (Lab M) and Christensen's urea broth (Southern Group, UK) were therefore set up in parallel for comparison. The results remained the same as those obtained after the first test. The table shows the results of the 13 isolates obtained by the three methods. Christensen's urea broth and the Micro Scan panels gave identical results in 12 of the 13 isolates tested, and the Lab $M$ and Micro Scan ornithine tests gave identical results for all the 13 isolates. The urease results obtained by the Minitek system differed from those obtained by Micro Scan panels and Christensen's urea broth for five isolates, and the ornithine results differed from those obtained by panels and the Lab M test for eight isolates.

\section{Discussion}

Using the $\mathrm{X}$ and $\mathrm{V}$ factor discs on nutrient agar plates, all the 98 isolates were initially found to be $\mathrm{V}$ dependent alone. Three of them, however, were subsequently identified as $H$ influenzae by both kits. This problem has been described by Kilian, ${ }^{2}$ who suggests using the porphyrin test to overcome the uncertainty of whether complex medium is totally free of $\mathrm{X}$ factor. For the biotyping of $H$ parainfluenzae, indole, ornithine, and urease are the key reactions. The two kits gave similar identification results for 77 of the 98 isolates examined after a single test. After repeat test the mismatching of biotypes obtained by the two kits remained. Back and Oberhofer examined 75 isolates of $H$ influenzae and 12 of $H$ parainfluenzae and found that Minitek gave comparable results. ${ }^{4}$ They did not give details of the conventional methods that were presumably used to biotype these isolates for the comparison to be made. With the 13 isolates (table), we examined the three methods simultaneously using the same cultures. The results given by Micro Scan correlated well with those obtained by the Christensen's urea broth method $(92 \%)$ and the Ornithine test by Lab M (100\%). The corresponding figures for the Minitek system were $61 \%$ and $30 \%$, respectively. We therefore decided to use the Micro Scan for all the subsequent work in our study. Furthermore, the colour change of the reactions in the Micro Scan panels was clear-cut. To obtain the complete test results, the Micro Scan panels required four hours of incubation; the Minitek system required 48 hours.

Commercially available kits are increasingly used by laboratories carrying out routine work. There are often several varieties available for the identification or biotyping of isolates. Sometimes it is difficult to decide which commercial system is the best available for the particular problem at hand. For our survey on the genital and respiratory carriage rates of different biotypes of $H$ parainfluenzae in men, we used the Minitek system as suggested in the Manual of Clinical Microbiology. ${ }^{2}$ It was based on results obtained by Back and Oberhofer, ${ }^{4}$ who studied only a small number of isolates of $H$ parainfluenzae. The results of our study indicate that further assessment of the Minitek system for the biotyping of the species is necessary. More studies with the recently introduced Micro Scan system are also required to confirm its reliability.

1 Kilian M. A taxonomic study of the genus Haemophilus, with a proposal of a new species. J Gen Microbiol 1976; with a propd

2 Kilian M. Haemophilus. In: Manual of clinical microbiology. 4th Wed 4th ed. Washington, DC
biology, 1985:387-93.

3 Houang E, Philippou M, Ahmet Z. Comparison of genital and respiratory carriage of Haemophilus parainfluenzae in men. J Med Microbiol 1989;26:119-23.

4 Back AE, Oberhofer TR. Use of the Minitek system for biotyping Haemophilus species. J Clin Microbiol 1978;7: 312-3. 